\title{
VOLUME OF FLUID BASED MODEL OF HEAVY FUEL OIL DROPLET EVAPORATION AND COMBUSTION
}

\author{
Paolo Guida* \\ Clean Combustion Research Center \\ KAUST \\ Thuwal, Mekkah 23955 \\ Email: paolo.guida@kause.edu.sa
}

\author{
Saumitra Saxena \\ Clean Combustion Research Center \\ KAUST \\ Thuwal, Mekkah 23955 \\ Saudi Arabia \\ saumitra.saxena@kaust.edu.sa
}

\author{
William L. Roberts \\ Clean Combustion Research Center \\ KAUST \\ Thuwal, Mekkah 23955 \\ Saudi Arabia \\ william.roberts@kaust.edu.sa
}

\begin{abstract}
The spray formation precedes combustion in all practical burners and constitutes the critical stage, which eventually determines the efficiency of the combustion process and level of emissions. Modern CFD simulations of combustion resolve simplified sub-models for droplet evaporation and combustion within a Lagrangian framework. Break-up effects like puffing and microexplosions are usually neglected but they eventually influence the evaporation and combustion behavior of heavy fuel sprays. We are developing a Volume of Fluid (VoF)-based CFD solver that allows us to model single droplet differential evaporation with the break-up effects. Puffing/micro-explosion and droplet ejection proceed in three steps: nucleation of a bubble of a light component within the droplet, expansion/coalescence of the bubbles and finally eruption with sub-droplets formation. Our goal is to individually model each event and then combine them in a composite simulation. Henceforth we can get data in realistic conditions to be used in Lagrangian spray simulations. We identified three relevant features which are necessary to create a reliable representation: interface tracking, differential evaporation, and compressibility effect. The solver is based on the Volume of Fluid (VoF) technique and coded within the open-source OpenFOAM framework. VoF technique consists in transporting the volume fraction of one of the two phases (liquid or gas). The NavierStokes equations are solved for a single-phase but adapting the
\end{abstract}

\footnotetext{
*Address all correspondence to this author.
}

physical properties to the volume fraction value. A state-of-theart method called iso-Advector is used to reconstruct the interface from the volume fraction field. The evaporation has been implemented as a source term in the volume fraction equation, and the conservation equations have been modified accordingly. In order to calculate the vapour and liquid physical properties, we implemented RKS equation of state (EOS). The droplet is assumed to have 2 phases: light and heavy, having physical properties comparable to diesel and heavy fuel oil (HFO), respectively. The pressure closure equation has been modified to handle large pressure differences during internal evaporation of light component. The validation of the solver is performed through benchmark cases as multiphase shock-tube, droplet oscillation and boiling interface either with experimental works and analytical solutions. Single suspended droplet experiment was performed to measure the velocity of an ejected micro-droplet during puffing using a shadowgraphy technique. The code is able to predict ejection velocity within a $15 \%$ error, which seems to be promising. The present article documents part of the algorithm development and its validation for the aforementioned methodology.

\section{INTRODUCTION}

Combustion of heavy fuels will play an important role in the future of energy generation. In the vast majority of the industrial applications, heavy fuels are burnt in the form of spray. 
The combustion of liquid fuels is a multi-physics and multi-scale phenomenON, involving complex chemistry, phase change and liquid interface evolution. Most of the models available nowadays for the simulation of spray burners and, in general, spray systems are based on a Lagrangian approach, where the droplets are modelled by adding a source term in the transport equations in correspondence of the position in the computational domain [1-3]. Proper sub-grid models become therefore of fundamental importance in order to quantify the impact of droplet-related variables of interest in time. However, the availability of reliable sub-grid models for heavy and in general multi-phase fuels is scarse. Most of the available codes rely on the so called $d^{2}$ law which predicts the decay of a ideal droplets in micro-gravity conditions. The $d^{2}$ law [4] and its various declinations [5] do not consider the highly non-ideal behavior that characterize heavy fuels. When a multi-component fuel, characterized by a wide boiling range, is exposed at high temperature, the formation of bubbles occur within the liquid phase. This happens because the mass transfer is slower than the temperature increase (high Lewis number). Those bubbles eventually expand as a consequence of the internal boiling until the pressure they exert on the droplet surface overcome the surface tension and an ejection takes place. Depending on the size of the bubbles both puffing or micro-explosions may take place. The puffing is defined as the formation of a ligament of fluid after the ejection of some amount of vapors. A micro-explosion is a disruptive event that leads to secondary atomization of the fuel droplet in several subdroplets. Those events are desirable since they increase the exposed surface which leads to an enhanced phase change and complete combustion. Predicting or eventually quantify those events is a difficult task. Few attempts were performed in the past to address such problems. Sazhin [6] tried to analytically identify the occurrence of micro-explosions while Watanabe [7] created a model to describe micro-explosions of emulsified fuels and was able to implement such model into a lagrangian framework to obtain a simplified but large scale simulation. However, the more sofisticated attempt to model puffing and micro-explosions was performed by Shinjo et al. [8]. He describes the phenomenon by utilizing the Volume of Fluid (VoF) approach coupled with the Level-Set method in order to obtain an accurate evolution of the interface. The model developed by Shinjo is able to resolve the ejection of vapors from a droplet due to the increase of internal pressure. It is difficult to validate a model with that level of complexity but the results obtained look promising. Another important problem regards the understanding and scaling of the observation related to droplet combustion. The experimental work performed on single droplets is, in fact, mostly based on single suspended droplet and falling droplet. Those experiments, even if particularly useful and widely used, do not represent real droplets in operating conditions. An industrial nozzle produces droplets in the order of tens on nanometer which is relevantly smaller than what it is achievable with single droplet experiments (hundred microns).

The goal of this work is to create a reliable CFD tool to qualitatively and quantitatively estimate the breakup induced by puffing and micro-explosions and its effect on combustion behavior. In order to do so, a new solver based on the VoF technique is currently under development. In this paper we describe the approach used and demonstrate the first results obtained so far.

The whole work is performed within the OpenFOAM framework the version 1812 from ESI was used.

\section{METHODOLOGY}

The VoF is a common methodology utilized to simulate multi-phase flows based on resolving a transport equation for the volume fraction of the liquid phase which is then used to assign the physical properties in the whole domain. The computational domain is then assumed to be one single phase but having different physical properties depending on the presence of liquid or gas. The volume fraction $\alpha$ is transported according to the following:

$$
\frac{\partial \alpha_{L}}{\partial t}+\nabla \cdot\left(\mathbf{u} \alpha_{L}\right)=\alpha_{L} \alpha_{G} \Gamma+\alpha_{L} \nabla \cdot \mathbf{u}
$$

The physical properties are averaged within the cell applying a simple volume fraction based average:

$$
\phi=\alpha_{L} \phi_{L}+\alpha_{G} \phi_{G}
$$

The Volume of Fluid has the main advantage of being intrinsically mass conservative under the assumption of incompressible flow. When dealing with bubble formation and bursts, this assumption becomes not reasonable. The terms on the right side of Eqn. 1 represent in fact the compressibility of the gas phase, and $\Gamma$ in particular is defined as:

$$
\Gamma=\frac{1}{\rho_{G}} \frac{D \rho_{G}}{D t}
$$

The interface is advected geometrically using the isoAdvector tool and the source terms integrated in the advection description. A sharp description of the interface is clearly fundamental to properly describe the evolution of the droplet interface. Moreover, the calculation of the normal to the interface needs to be accurate to properly quantify the surface tension force which is modelled according to Brackbill et al. [9]:

$$
f_{s}=\sigma \mathbf{k} \nabla \alpha
$$


where $\sigma$ is the surface tension coefficient and $\mathbf{k}$ the curvature expressed as the normalized gradient of the volume fraction. The coupling between momemntum and pressure is solved by adopting the PIMPLE approach which is an hybrid methodology between the well known PISO and SIMPLE iterative procedures. The momentum equation and an equation for pressure based on the continuity are solved subsequently until convergence is achieved.

$$
\begin{gathered}
\frac{\partial \rho \mathbf{u}}{\partial t}+\nabla \cdot(\rho \mathbf{u u})=\nabla \cdot \mu(\nabla \mathbf{u})-\nabla p+f_{s} \\
\frac{\partial \rho}{\partial t}+\nabla \cdot(\rho \mathbf{u})=0
\end{gathered}
$$

The physical properties of both liquid and gas phase are calculated with the Peng-Robinson equation of state which covers a wide range of operative conditions and will be useful when increasing the complexity of the solver by adding species and thermodynamic pseudo-equilibrium at the contact interface between the two phases.

\section{VALIDATION}

Four cases have been chosen as benchmark for the validation of the code. The choice of the benchmarks was driven by the main needs of the solver. It is very important to be able to predict extreme cases since the availability of detailed experiment on idealized conditions describing the phenomenon is poor. The first test case is the calculation of the spurious currents in a static configuration. In order to do so a simulation was performed on the static case reported by Fuster et al. [10]. The maximum amplitude of the velocity fluctuations observed within a time-frame of $1 \mathrm{~s}$ is $1 \mathrm{E}-10 \mathrm{~m} / \mathrm{s}$ which is considered satisfactory since such velocity is not expected to play a big role in the actual numerical simulation.

\section{Air-Water Shock Tube}

The second case consists in a water-air shock tube. It is very important for the solver to be able to follow a shock generated by strong pressure gradients and density gradients in order to predict the burst of a bubble. It is useful to notice that the physics associated to micro-explosions is close to that of underwater explosions. A 2-phase 1-D shock-tube having water on the right side and air on the left side is set up. The discontinuity in volume fraction and other variables is present in the middle of the domain. The boundary conditions are open since the domain is large enough to guarantee propagation without touching the walls. A list of the initial conditions in the domain is presented in Tab 1.
TABLE 1. WATER-AIR SHOCK INITIAL CONDITIONS.

\begin{tabular}{cll}
\hline Variable & Left $(x<5)$ & Right $(x>5)$ \\
\hline $\mathrm{U}$ & $0 \mathrm{~m} / \mathrm{s}$ & $0 \mathrm{~m} / \mathrm{s}$ \\
alpha & 1 & 0 \\
$\mathrm{p}$ & $1 \mathrm{e}+9 \mathrm{~Pa}$ & $1 \mathrm{e}+5 \mathrm{~Pa}$ \\
$\mathrm{~T}$ & $308 \mathrm{~K}$ & $308 \mathrm{~K}$ \\
\hline
\end{tabular}

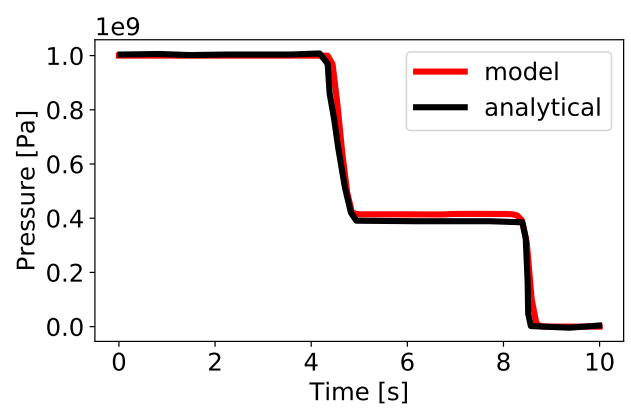

FIGURE 1. COMPARISON OF PRESSURE PROFILE BETWEEN MODEL PREDICTION AND ANALYTICAL SOLUTION OF A TWO PHASE AIR-WATER SHOCK TUBE.

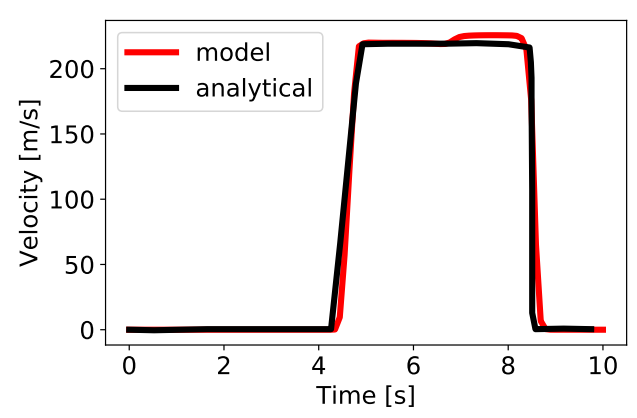

FIGURE 2. COMPARISON OF VELOCITY PROFILE BETWEEN MODEL PREDICTION AND ANALYTICAL SOLUTION OF A TWO PHASE AIR-WATER SHOCK TUBE.

Oscillating Droplet The third case that was examined is an oscillating droplet. The use of this kind of benchmark was suggested by the work of Shinjo et al. [8]. It consists in releasing a droplet with the shape of a prolate ellipse in a zero-gravity domain and let it assume gradually the spherical shape driven by surface tension. This benchmark is usefull to understand whater the surface tension is acting properly and the interface reconstructed nicely. Figure 5 shows some of the frames obtained from 
the simulations. The analytical result is not reported here for simplicity but it is possible to refer to [8]. Again the computational domain is $2 \mathrm{D}$ and the solution is compared to the analytical solution given an error lower than 1e-3 in the fluctuation therm $\omega$.

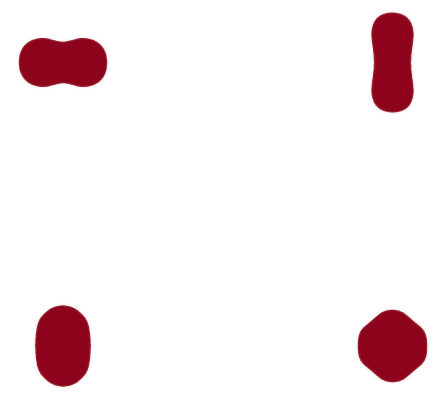

FIGURE 3. SURFACE TENSION DRIVEN OSCILLATIONS OF A DEFORMED DROPLET IN MICRO-GRAVITY.

\section{Boiling}

In order to deal with the mass transfer during the internal evaporation, a new source term was included in the classical alpha equation. The equation reads now:

$$
L S=\alpha_{L} \alpha_{G} \Gamma+\alpha_{L} \nabla \cdot \mathbf{u}-\alpha_{L} \dot{m}\left(\frac{1}{\rho_{L}}-\frac{1}{\rho_{G}}\right)
$$

The pressure needed to be updated as well since it involves the continuity equation which is influenced by mass transfer. The mass transfer is calculating by considering a boiling interface and therefore no diffusion based evaporation is present. The mass transfer is simply calculated as follow:

$$
\dot{m}=\left(\frac{k \nabla(T)}{\Delta H_{v} a p}\right) \nabla(\alpha)
$$

This simplification is justified from the fact that boiling is the phenomenon that drive puffing and micro-explosions more than pure evaporation. The code is validated against a classical "sucking interface problem" following again the setup of Shinjo et al. [8]. Really good agreement is once again observed between analytical and numerical solutions. It is important to point out

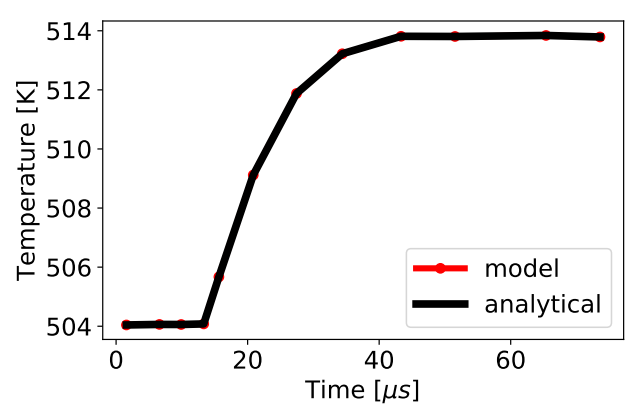

FIGURE 4. 1D BOILING PROBLEM, TEMPERATURE COMPARISON.

that this case does not consider interactions of interface deformation and boiling which may result in numerical problems and unphysical solutions. It is therefore recognized as necessary a different set of tests which is not performed in this work but that will be objective of future studies.

\section{RESULTS}

In this section the solver is tested on a real case but appropriately simplified. The idea is to replicate the ejection of a fuel particle of an emulsified Heavy Fuel Oil 380 (HFO) droplet. HFO emulsions are of particular interest because the presence of water within a high-boiling fuel generates a very intense atomization mechanism which leads the production of several sub-droplets. The experimental data are obtained from a suspended droplet facility. More information on the experimental setup and other conditions can be found in the following reference [11]. The experiment, which is strongly non-ideal, is simplified by assuming the gravity negligible, which is a valid assumption when dealing with high surface tension fluids. The temperature profile is imposed as the initial condition on the whole computational domain and it is equal to $650 \mathrm{C}$ in the bulk (which we are assuming to be vapor) and $380 \mathrm{~K}$ within the droplet (measured by thermocouple during the experiment). The mesh is $2 \mathrm{D}$ and it counts $200 \times 200$ cells. the pressure is atmospheric in the bulk. A bubble is initialized within the fuel droplet having the physical properties of water vapor and its size was calculated according to the initial size of water droplets within the emulsion and estimated to be $100 \mathrm{~nm}$. The pressure within the bubble was calculated according to the Young-Laplace equation:

$$
\Delta P \approx \frac{2 R}{\sigma}
$$

where $\sigma$ is the surface tension coefficient and $\mathrm{R}$ the radius of the bubble, $\Delta P$ is the pressure difference between the liquid bulk and the bubble. The physical properties of the HFO were measured 
in previous works. The surface tension coefficient is assumed to be similar to that of n-eicosane and obtained from the Yaws handbook (this is a reasonable assumption when at high temperature). The ejection of 2 small droplets is observed after 1E-5 s. The droplets travel at a velocity of approximately $0.5 \mathrm{~m} / \mathrm{s}$ which is very similar to what observed in the experimental case at the same conditions $(0.45-0.55 \mathrm{~m} / \mathrm{s})$. A more detailed and fine mesh

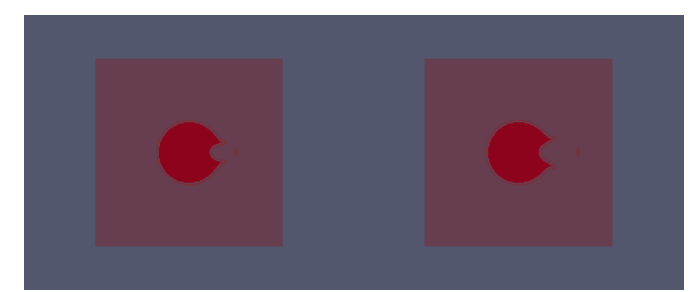

FIGURE 5. DROPLET EJECTION IN 2D SIMULATION.

is expected to strongly improve the solution, moreover, mesh independence tests will be performed in the future.

\section{CONCLUSIONS AND OUTLOOK}

A CFD model for the description of puffing and microexplosions is under development. We have proved that the code is able to handle drastic pressure gradients (shock tube), interface oscillations and a simplified boiling. The code will be further modified in the future to have a better description of the whole evaporation process (diffusion based) and it will be tested on more complex mesh topology. The ultimate scope of this work is to have a comprehensive solver that can describe, nucleation growth and ejection from where develop reduced order models for large CFD applications. Another scope of the work is to introduce reactivity and eventually study the effect of puffing/microexplosions on flame stability, efficiency of combustion and emissions.

\section{ACKNOWLEDGMENT}

The research reported in this publication was supported by the King Abdullah University of Science and Technology (KAUST).

\section{REFERENCES}

[1] Zaripov, T. S., Gilfanov, A. K., Begg, S. M., Rybdylova, O., Sazhin, S. S., and Heikal, M. R., 2017. "The fully lagrangian approach to the analysis of particle/droplet dynamics: Implementation into ansys fluent and application to gasoline sprays". Atomization and Sprays, 27(6).
[2] Hu, Y., Olguin, H., and Gutheil, E., 2017. "A spray flamelet/progress variable approach combined with a transported joint pdf model for turbulent spray flames". Combustion Theory and Modelling, 21(3), pp. 575-602.

[3] Desantes, J. M., Garcia-Oliver, J. M., Pastor, J. M., and Pandal, A., 2016. "A comparison of diesel sprays cfd modeling approaches: Ddm versus $\sigma-\mathrm{y}$ eulerian atomization model". Atomization and Sprays, 26(7).

[4] Lee, A., and Law, C. K., 1992. "An experimental investigation on the vaporization and combustion of methanol and ethanol droplets". Combustion Science and Technology, 86(1-6), pp. 253-265.

[5] Hallett, W. L., 2000. "A simple model for the vaporization of droplets with large numbers of components". Combustion and Flame, 121(1-2), pp. 334-344.

[6] Sazhin, S., Rybdylova, O., Crua, C., Heikal, M., Ismael, M., Nissar, Z., and Aziz, A. R. B., 2019. "A simple model for puffing/micro-explosions in water-fuel emulsion droplets". International Journal of Heat and Mass Transfer, 131, pp. 815-821.

[7] Watanabe, H., Matsushita, Y., Aoki, H., and Miura, T., 2010. "Numerical simulation of emulsified fuel spray combustion with puffing and micro-explosion". Combustion and flame, 157(5), pp. 839-852.

[8] Shinjo, J., Xia, J., Ganippa, L., and Megaritis, A., 2014. "Physics of puffing and microexplosion of emulsion fuel droplets". Physics of Fluids, 26(10), p. 103302.

[9] Brackbill, J., Kothe, D., and Zemach, C., 1992. "A continuum method for modeling surface tension". Journal of Computational Physics, 100(2), pp. $335-354$.

[10] Fuster, D., and Popinet, S., 2018. “An all-Mach method for the simulation of bubble dynamics problems in the presence of surface tension". Journal of Computational Physics, 374, Dec., pp. 752-768.

[11] Jiang, L., Elbaz, A. M., Guida, P., Al-Noman, S. M., AlGhamdi, I. A., Saxena, S., and Roberts, W. L., 2019. "Cenosphere formation during single-droplet combustion of heavy fuel oil”. Energy \& fuels, 33(2), pp. 1570-1581. 